\title{
Ecodesign assessment information an important tool for the design of new elements for building construction
}

\author{
González-Madariaga, Francisco J. ${ }^{\text {a' }}$ Rosa-Sierra, Luis A. ${ }^{\text {b \& Gómez Gómez, Jaime F. }}$ \\ ${ }^{a}$ PhD Full Professor. CUAAD. Universidad de Guadalajara, México. francisco.madariaga@cuaad.udg.mx \\ ${ }^{\text {b} P h D ~ F u l l ~ P r o f e s s o r . ~ C U A A D . ~ U n i v e r s i d a d ~ d e ~ G u a d a l a j a r a, ~ M e ́ x i c o . ~ a l b e r t o . r o s s a @ c u a a d . u d g . m x ~}$ \\ ${ }^{\mathrm{c}} \mathrm{PhD}$ Full Professor. CUAAD. Universidad de Guadalajara, México.jaime.gomez@cuaad.udg.mx
}

\begin{abstract}
Resumen
En el proyecto que se entrega, se busca demostrar la Hipótesis que señala que es posible la fabricación de elementos constructivos, partiendo de residuos de partículas de plásticos expandidos y fibra de agave como agregados, todos conglomerados en una pasta de yeso. Se ha llevado a cabo un proceso investigación de materiales, de diseño y desarrollo de producto, que propone la aplicación de los resultados de este proyecto de investigación, en un sistema constructivo para particiones interiores y techos falsos. Una de las metas principales para este proyecto es la de ofrecer un elemento constructivo con menor impacto medioambiental. Aqui se muestran algunos avances sobre la valoración de impacto ambiental del nuevo sistema constructivo y que permiten observar una mejoría, comparado con algunos sistemas ya disponibles.
\end{abstract}

Palabras clave: Ecodiseño, Reciclaje del plástico, Elementos de construcción

\begin{abstract}
A new wallboard panel has been developed. The flat panel is manufacturated mainly with a nucleus of gypsum plaster and enriched with agave dry fiber and expanded plastic particles, both agave and plastic are recovered materials and they cooperate to produce a light and resistant flat building construction product. Wallboards samples were produced according several formulas and under different conditions, then they were tested in materials labs. Today most of project stages are close to be finished and the research team works on a whole new building construction system design. One of the main objectives for this project is to reach a better ecological impact than other similar systems, in order to verify those benefits an Ecodesign assessment has been carried on. In this paper some important information and data about the assessment are shown.
\end{abstract}

Keywords: Ecodesign, Plastics recycling, Building construction elements 


\section{Desarrollo}

\subsection{Diseño del Proyecto de Investigación}

El proyecto de investigación que se muestra: Diseño de elementos para un sistema constructivo basado en innovadoras placas fabricadas con yeso, fibra de agave y partículas de plástico expandido; incluye 6 fases:

(1) Antecedentes, (2) Trabajos previos, (3) Etapa experimental I, (4) Etapa experimental II, (5) Diseño y desarrollo de producto y, (6) Evaluación de ecodiseño.

\section{Hacia la disminución del impacto de los productos al medioambiente. El ecodiseño.}

El Ecodiseño analiza las relaciones que mantienen el medioambiente y un producto a lo largo del ciclo de vida de este último, al tiempo busca que las funciones propias del producto sean las más eficientes. La mayoría de los objetivos del ecodiseño sólo se pueden lograr si son incorporados durante la fase de diseño. Una herramienta valiosa para mejorar el desempeño ambiental de productos es la Evaluación de ecodiseño (Rieradevall, 1999).

\section{Trabajos previos de investigación}

Los trabajos previos del proyecto (fases 1 y 2 ) permitieron analizar información de dos de los componentes usados para la fabricación de placas; yeso y partículas de plástico expandido. Durante las fases se obtuvieron datos del amasado de pastas y de la integración de los residuos de plástico en esas pastas. Se determinaron proporciones de los componentes, viscosidad de las pastas, tiempos de fraguado, endurecimiento, y densidades aparentes obtenidas.

\section{Etapa experimental 1}

\subsection{Selección de mezclas}

La etapa experimental 1 dio inicio con el diseño de un árbol de combinaciones de los diferentes materiales organizados en grupos de trabajo, en total 68 mezclas, y de todas ellas se produjeron muestras. Además, se fabricaron dos grupos de muestras que no incluyen partículas de plástico, y que funcionaron como materiales de comparación. Todas las muestras $(12.5 \times 300 \times 400 \mathrm{~mm})$ se recubrieron con papel grueso en sus 2 caras y fueron sometidas a los siguientes ensayos: absorción de agua por inmersión total, determinación de densidad laminar, ensayo de flexión, ensayo de reacción al ataque directo de flama y resistencia al impacto. Las normas empleadas, los procedimientos detallados y los resultados de los ensayos se encuentran disponibles en otras publicaciones (González, Madariaga: 2006)

\section{Etapa Experimental 2}

\subsection{Fabricación de nuevas probetas}

En la Etapa Experimental 2, se seleccionó y fabricó un nuevo grupo de muestras que posteriormente fueron sometidas a ensayos para su caracterización. Para mejorar su desempeño a esfuerzos de flexión, las mezclas incluyen ahora un nuevo componente: fibras cortas de agave. 
Las normas empleadas, los procedimientos y resultados detallados de los ensayos se encuentran disponibles en otras publicaciones (González Madariaga: 2012a) .

\subsection{Caracterización. Aplicación de usos similares a la placa de yeso estándar}

Los ensayos para caracterización de placas son los mismos en las dos etapas experimentales (punto 4.1), esto permite establecer una comparación entre ambos trabajos de laboratorio. Los resultados de los ensayos se resumen así:

\subsubsection{Resultados de los ensayos}

\subsubsection{Ensayo de absorción al agua por inmersión total de las placas}

De los datos se puede extraer que se obtuvieron resultados favorables en donde las muestras registraron un menor porcentaje en absorción de agua, lo que nos indica que la inclusión del plástico EPS como segundo material, ayuda a disminuir los niveles de absorción. Sin embargo esta característica disminuye con la presencia de fibra de agave donde se da una mayor absorción de agua.

\subsubsection{Ensayo de Densidad Laminar.}

La densidad laminar es la cantidad de masa de una placa en función de su espesor $\left(\mathrm{kg} / \mathrm{m}^{2}\right)$. La serie de muestras que incluyen partículas de plástico presentó una densidad laminar menor que aquellas muestras sin partículas. Por su parte las muestras que tienen agregados de fibra en su composición reportaron masas competitivas comparadas con productos similares disponibles en el mercado, de aquí se puede concluir que la composición que se necesita para obtener los resultados deseables para el proyecto se obtienen de un balance de ambos grupos: si bien la presencia de partículas de EPS disminuye la densidad de los nuevos materiales hasta en un $26 \%$, la fibra de agave en placas produce un aumento de masa. Sin embargo el uso de fibras se justifica ya que ofrece otros beneficios, como el aumento de resistencia a la flexotracción.

\subsubsection{Ensayo de Flexión de las placas}

Estos ensayos se llevaron a cabo bajo la dirección del Dr. Francisco Javier Talavera investigador del Instituto de Celulosa y Papel del Centro Universitario de Ciencias exactas e Ingeniería (CUCEI) de la Universidad de Guadalajara, México. Los resultados del ensayo aún se analizan, pero se dispone ya de algunas conclusiones: el grupo de placas que presentó menor resistencia a la flexión fue el grupo de placas fabricadas con pastas de yeso, residuos de EPS y agua. El grupo de placas con agregados de fibra de agave presentó una resistencia mayor a la flexotracción, esto parece confirmar que los agregados de fibra de agave favorecen la resistencia de las placas a estos esfuerzos.

\subsubsection{Ensayo de Reacción al Fuego.}

De los ensayos sobre placas se obtiene que las muestras obtenidas de placas comerciales presentaron una afectación por ataque de fuego directo muy similar a las placas de la investigación. El grupo de placas con Yeso, Agua, Fibra y EPS que presentó un comportamiento desfavorable en los bordes fueron las de la serie YEP con un promedio de $10 \mathrm{~cm}^{2}$ de área afectada, mientras que las que resultaron más favorables para éste ensayo fueron las placas $\mathrm{YEP} / \mathrm{E}$ que su área afectada es $50 \%$ menor que las mencionadas anteriormente. Esto nos habla no sólo de la importancia de la cantidad de fibras de agave en las placas, sino también de la forma como se originaron las fibras empleadas. Es conveniente recordar que las fibras de agave integradas en las placas, son residuos del proceso de obtención de la bebida alcohólica denominada tequila. En la industria del tequila hay dos procesos de prensado para la obtención de mieles 
para la destilación; prensado del agave en crudo y otro prensado después de ser sometido a cocción. En esta investigación se emplean fibras obtenidas por ambos procesos.

\subsubsection{Ensayo de Resistencia al impacto de las placas}

En los resultados preliminares (los datos más precisos aún se encuentran en proceso de publicación) se observa que sólo un grupo de placas superó los límites marcados por la Norma. En la mayoría de los casos, las placas que contienen fibra de agave muestran resultados favorables para el proyecto, de aquí se puede expresar que la presencia del agregado de agave en las placas investigadas, mejora las propiedades de resistencia al impacto en las muestras donde participa

\subsection{Conclusiones de la Etapa Experimental 2}

Una ventaja principal de los nuevos materiales de placa está en su ligereza, donde una placa de 12,5 $\mathrm{mm}$ de uno de los nuevos materiales tiene un peso de hasta $5,55 \mathrm{~kg} / \mathrm{m}^{2}$ mientras una placa comercial tiene su peso entre 9,8 y $10 \mathrm{~kg} / \mathrm{m}^{2}$. La ventaja disminuye con la inclusión de fibras de agave, sin embargo se puede decir que esta característica facilita su manejo en producción y que puede traducirse en un beneficio medioambiental. También se añade como beneficio medioambiental la aplicación de productos revalorados (como las partículas de plástico y la fibra de agave) en las nuevas placas de materiales reciclados.

Los ensayos han confirmado las buenas características de reacción al fuego de los materiales propuestos. En varias de las comparaciones entre las resistencias mecánicas de las placas estándar y las placas fabricadas con residuos de espuma y fibra se observa un mejor desempeño de los materiales comerciales, la pérdida de resistencia en los nuevos materiales se puede explicar por la rica proporción de agua empleada en la preparación de las mezclas y a la utilización de residuos de EPS lo que resulta en una estructura ligera, pero de alta porosidad y por ello más débil.

Del grupo de ensayos aplicados a probetas de placas, se confirma que los nuevos materiales son utilizables en aplicaciones similares a las de placas estándar de yeso laminado. Sin embargo es menor la resistencia de las placas investigadas.

Se ha mostrado que las aplicaciones propuestas son una alternativa para la revaloración de residuos de espumas de EPS y fibras de agave. Se ha logrado así una propuesta de productos alternativos para la construcción, que además pueden fabricarse con procesos accesibles para una pequeña o mediana empresa.

\section{Evaluación comparativa de Ecodiseño}

La evaluación de Ecodiseño (en adelante ECC) es un proceso que aplica diversas herramientas de recolección de información y análisis, los resultados son especialmente útiles en la toma de decisiones durante el proceso de diseño. En este escrito, se enfatiza en la disminución de los requerimientos de energía y materiales, así como el manejo de residuos durante todas las etapas del ciclo de vida de un producto específico (González M. 2012b).

\subsection{Etapas del proceso de Ecodiseño}

Las etapas generales de Evaluación de Ecodiseño incluyen :

A. Creación del equipo y planificación, B. Evaluación de ecodiseño, C. Implantación de las mejoras determinadas en la evaluación, D. Seguimiento de las mejoras, E. Valoración del proyecto. La etapa B, incluye las siguientes acciones: 


\subsubsection{Descripción del producto}

Identificación y organización de todos los elementos del producto a evaluar.

\subsubsection{Descripción de materiales}

Identificación de los materiales con los que está fabricado el producto por evaluar

\subsubsection{Descripción del sistema producto}

Esta etapa relaciona las cinco etapas del ciclo de vida de un producto con los materiales y procesos involucrados.

\subsubsection{Determinación de la unidad funcional}

De acuerdo a los intereses del proyecto se establece una cantidad de producto a evaluar. Es conveniente enfatizar que no se debe de confundir un lote con una unidad funcional, ya que ésta última se relaciona no sólo con la cantidad de materiales y energía requeridos.

\subsubsection{Aplicación de la matriz MET}

La matriz MET es una herramienta semicuantitativa de análisis de impacto medioambiental de producto, que permite organizar de manera sistemática la información ambiental relevante relacionada con la evaluación, lo que facilita al equipo de ecodiseño la posibilidad identificar amenazas y oportunidades de mejora. La matriz MET permite capturar y organizar en una tabla, información acerca de los materiales (M) utilizados, la energía (E) consumida y las emisiones tóxicas (T) generadas durante las diferentes etapas del ciclo de vida de un producto. La matriz MET favorece el análisis grupal.

\subsubsection{Análisis de la matriz MET}

De la elaboración, discusión y análisis de la matriz MET obtenida, el equipo de ecodiseño propone acciones generales de mejora.

\subsubsection{Ecoperfil de producto}

Con la información generada el equipo de ecodiseño evalúa el desempeño del producto. La gráfica esta compuesta por seis vectores: a. satisfacción de las funciones que debe cumplir el producto, b. eficiencia de la selección de materiales en los que está fabricado, c. fabricación del producto, eficiencia en la selección y aplicación de las técnicas de producción a través de las cuales se obtiene el producto, d. comercialización y distribución, descripción y evaluación del proceso que lleva el producto evaluado de su fabricante al usuario del mismo, e. Uso del producto, el equipo evalúa como se usa el producto e identifica oportunidades de mejora, f. eliminación final o disposición, la forma como el producto finaliza su ciclo de vida es también una fuente de oportunidades de mejora. La evaluación se practica por el equipo mediante la asignación de una calificación numérica relativo a la satisfacción que ofrece el producto en los seis vectores, finalmente los resultados se unen formando una gráfica poligonal.

\subsubsection{Aplicación de ecoindicadores}

Los materiales necesarios para la producción de la unidad funcional se relacionan aritméticamente con ecoindicadores. Los ecoindicadores son valores sin dimensión que cuantifican el impacto ambiental de un material y/o proceso de producción específicos.

\subsubsection{Tablas de Ecoindicadores, producción , uso y disposición}

En una tabla se relacionan las cantidades obtenidas para cumplir con la unidad funcional con el ecoindicador correspondiente (IHOBE:1999), esto resulta en un indicador de desempeño ambiental 
expresado en $\mathrm{Mp}$ (milipuntos); el resultado, es un elemento de comparación útil para valorar una estrategia o acción de diseño con su desempeño medioambiental.

\subsubsection{Propuestas de mejora}

Con base a los resultados de evaluación obtenidos, el equipo de diseño propondrá estrategias de mejora al producto relacionándolas con cada etapa de su ciclo de vida.

\subsubsection{Viabilidad de las mejoras}

El equipo de ecodiseño califica las propuestas del punto anterior, atendiendo criterios como: sencillez técnica, factibilidad económica y otros de importancia particular de la estrategia.

\subsubsection{Propuesta de alternativas de mejora}

Se comparan entre sí las alternativas propuestas y sus resultados hipotéticos.

\subsubsection{Ecoperfil comparativo de resultados de ecodiseño}

Retomado la gráfica poligonal (ver 6.1.7), se evalúan y comparan los resultados obtenidos de la aplicación de las alternativas de mejora al producto, con relación al estado inicial del proyecto.

\subsubsection{Conclusiones de la evaluación}

\section{Desarrollo de la evaluación}

Una vez determinadas y caracterizadas las fórmulas de las nuevas placas, se practicó una evaluación ECC; dos mezclas de las ensayadas (mezclas claves 121 y 123) contra otra placa similar disponible en el mercado. La evaluación tiene como objetivo principal validar las mejores conductas de los nuevos productos hacia el medioambiente.

Nota importante: Aquí sólo se exponen; la figura 1 y la tabla 1. Quedan para futuras publicaciones la exposición de las restantes obtenidas en el proceso de ECC.

\subsection{Descripción del producto a evaluar}

En el mercado se encuentran tres tipos de placas de yeso comercial: Uno, el panel laminado de yeso adherido en sus caras a dos cartones delgados. Para mejorar la resistencia a la flexión, el panel de yeso estándar contiene fibra de celulosa en su núcleo. Dos la placa resistente al fuego: contiene un núcleo reforzado con la integración de fibra de vidrio resistente a medios alcalinos, la cual le proporciona características de resistencia al fuego, y; Tres, Placa de yeso para exterior, que está formado por un núcleo de yeso reforzado con agentes impermeables, que lo hacen resistente a la humedad.

\subsubsection{Principales fabricantes de placas de yeso en México}

USG. (United States Gypsum Company). KNAUF, Con una capacidad superior a 1000 millones de $\mathrm{m}^{2}$, KNAUF es la mayor fabricante de placas de yeso del mundo y por último, PLACA COMEX que nace de la fusión de dos industrias en México; la primera COMEX líder mexicano en la fabricación de pinturas y recubrimientos con 50 años en el Mercado y la segunda, LAFARGE, líder mundial en la industria de materiales para la construcción.

7.1.2 Proceso típico de fabricación de placas laminadas de yeso

Un proceso típico para la fabricación de placas de yeso se puede resumir asi: 
1.Trituración del Yeso, el yeso procedente de la cantera se reduce de tamaño, obteniéndose un producto con un tamaño máximo de $35 \mathrm{~mm}$, que es el adecuado para alimentar la molienda. 2.Molienda, el yeso es sometido a compresión, entre unos rodillos y un plato de molienda reducido su tamaño progresivamente hasta unas 200 micras, que es el adecuado para calcinarlo y posteriormente formar el yeso-escayola para la fabricación de las placas. 3. Calcinación, mediante la calcinación, proceso de calentamiento del yeso a $160^{\circ} \mathrm{C}$ durante unos $30 \mathrm{~min}$., el yeso adquiere la propiedad hidráulica, es decir amasada con agua la pasta endurece en minutos. 4. Línea de producción de placas, al yeso en polvo calcinado se le añade agua y aditivos con lo que se obtiene una pasta que fraguará en pocos minutos. Por medio de la maquinaria de la línea de producción, esta pasta de yeso se introduce de forma automática y en proceso contínuo, entre dos láminas de cartón, que lleva a la obtención de la placa de yeso laminado. Posteriormente esta placa se corta a la medida especificada, se seca en un horno y se embala. 5. Almacén y distribución.

\subsubsection{Proceso general de instalación de placas de yeso}

Los elementos que intervienen en la instalación de las placas son igualmente importantes pues forman parte del ciclo de vida del producto. En breve, el proceso consiste en colocar y asegurar las placas sobre una estructura metálica, la estructura generalmente está constituida por materiales livianos como postes y canales galvanizados.

\subsection{Descripción del sistema - producto}

La determinación del sistema-producto para la evaluación se decidió tomando considerando las etapas del Ciclo de Vida Simplificado (CVS) de un producto: Obtención de los materiales, Producción, Comercialización y distribución, Uso del producto y Fin de vida. Adelante (fig.1), se muestran los materiales considerados para valoración, relacionándolos con las etapas del CVS al mismo tiempo que se especifican los sub-procesos que han sido tomados en cuenta, en cada etapa del sistema-producto placa de yeso laminado de núcleo regular. De la información del fabricante, se tiene que el lote de producción se transportará de la planta de fabricación al centro de distribución, desde donde se surtirán los puntos de venta distribuidos en toda la República Mexicana.

Según el datos del fabricante la placa tiene una fase de vida útil de 30 años. En su fase de uso se incluye la instalación de la placa con todos los accesorios necesarios, como lo son: perfiles, tornillos, cinta y pasta para juntas.

\section{3 determinación de la unidad funcional}

Para efectos de este estudio se determinó que la unidad funcional será: una pared con un área de $2.4 \mathrm{~m} \mathrm{x}$ $3.6 \mathrm{~m}$ recubierta con placas de yeso de $12.5 \mathrm{~mm}$ de espesor, la evaluación comprende todos los materiales necesarios para su construcción, excepto acabados. 


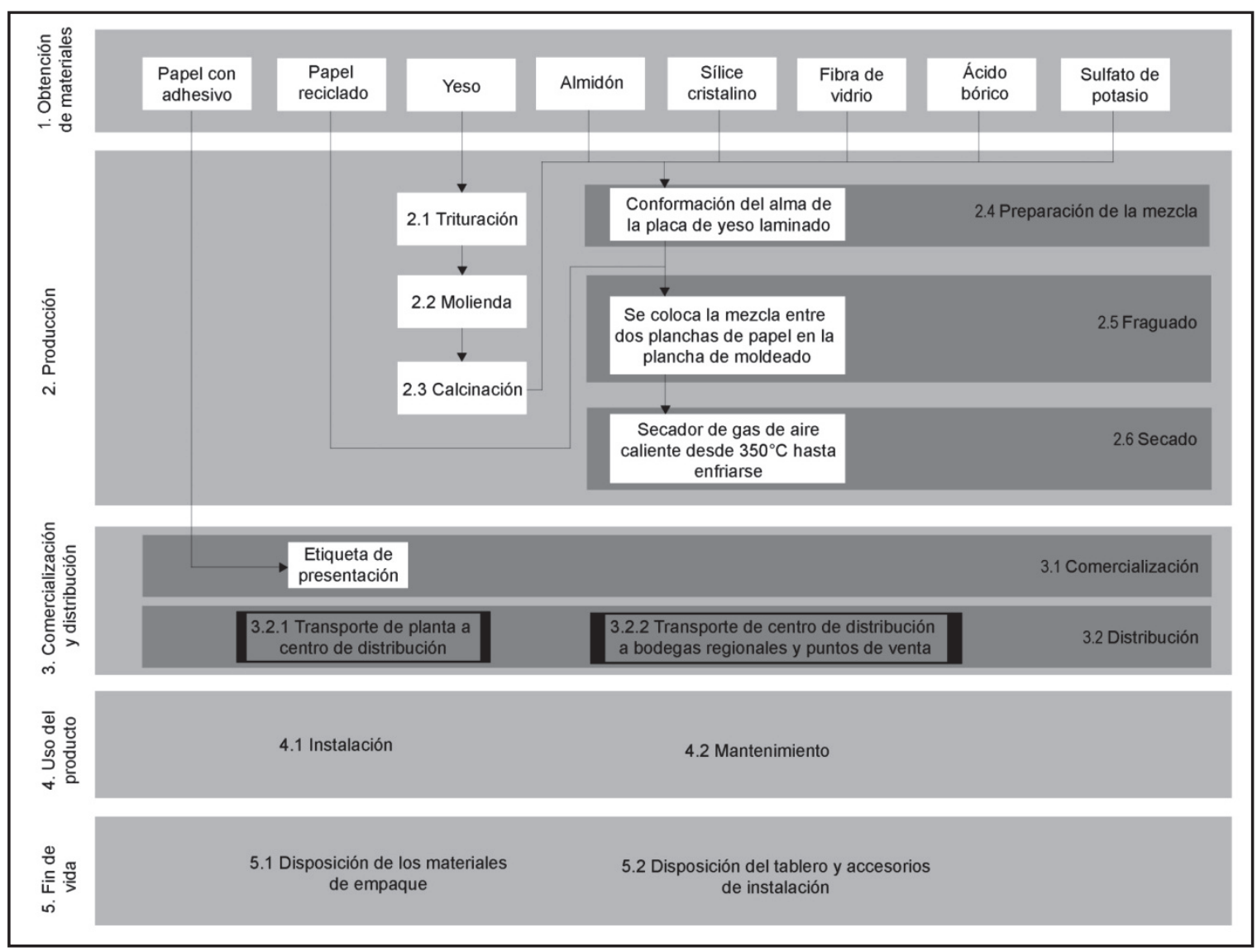

Fig 1. Sistema producto a evaluar . Fuente: Elaboración propia. Elaboración propia. G.Madariaga, Delsordo N. Selene, González Q. A. Patricia (2014)

\subsection{Matriz MET}

Se examinan los principales impactos detectados en la matriz MET (tabla1). Los impactos están asociados a las cinco fases del ciclo de vida del sistema-producto y se resumen así:

\subsubsection{Obtención de materiales}

Para la fabricación de las placas de yeso aligeradas, se necesitan 2 tipos de yeso, vulgar y fino, partículas de plástico expandido de entre 3 y $6 \mathrm{~mm}$ y fibra de agave seca. Se usa papel o cartón de reciclado para ambas las caras de la placa. Se requieren de distintos elementos accesorios como perfiles metálicos, pasta y cinta para poder su instalación. Todos los materiales se consideran en crudo, no se consideran para efectos de la evaluación los procesos de transformación previos 
González-Madariaga, Francisco J.; Rosa-Sierra, Luis A. \& Gómez Gómez, Jaime F.

Tabla 1 Matriz, met. Producto comercial

\begin{tabular}{|c|c|c|c|c|c|c|}
\hline Etapas & \multicolumn{4}{|c|}{ Impacto en los recursos materiales $(\mathrm{M})$} & Recursos (E) & Emisiones (T) \\
\hline \multirow{8}{*}{$\begin{array}{l}\text { 1. Obtención de materiales } \\
\text { Se consideran los materiales } \\
\text { crudos, pero no se consideran } \\
\text { todos los procesos de } \\
\text { transformación hasta } \\
\text { convertirse en los insumos } \\
\text { para la elaboración de la } \\
\text { placa de yeso laminado. }\end{array}$} & Yeso & Yeso & 59.2 & $\mathrm{~kg}$ & \multirow{8}{*}{$\begin{array}{l}\text { La energía } \\
\text { asociada a la } \\
\text { obtención y } \\
\text { transporte. }\end{array}$} & \multirow{6}{*}{$\begin{array}{l}\text { Emisiones de } \\
\text { gases } \\
\text { invernadero } \\
\text { asociadas a la } \\
\text { obtención y } \\
\text { transporte. }\end{array}$} \\
\hline & \multirow{5}{*}{ Químicos } & $\begin{array}{l}\text { Sulfato } \\
\text { potasio }\end{array}$ & 3.48 & $\mathrm{~kg}$ & & \\
\hline & & Almidón & 2.10 & $\mathrm{~kg}$ & & \\
\hline & & Sílice cristalino & 3.48 & $\mathrm{~kg}$ & & \\
\hline & & Fibra de vidrio & 0.70 & $\mathrm{~kg}$ & & \\
\hline & & Ácido bórico & 0.70 & $\mathrm{~kg}$ & & \\
\hline & & Papel reciclado & 10.4 & $\mathrm{~kg}$ & & Emisiones de \\
\hline & Celulosa & Cinta de papel & 7.32 & $\mathrm{~m}$ & & $\begin{array}{l}\text { clorados y } \\
\text { azufre. }\end{array}$ \\
\hline & 2.1 Trituración & Yeso natural & 59.2 & $\mathrm{~kg}$ & & \\
\hline & 2.2 Molienda & Yeso natural & 59.2 & $\mathrm{~kg}$ & & \\
\hline & 2.3 Calcinación & Yeso natural & 59.2 & $\mathrm{~kg}$ & & \\
\hline 2 Drodución & & Yeso calcinado & 59.2 & $\mathrm{~kg}$ & & \\
\hline 2. Produccion & & Almidón & 2.10 & $\mathrm{~kg}$ & & \\
\hline Se desglosan materiales y & & Sílice cristalino & 3.48 & $\mathrm{~kg}$ & La energía & material, \\
\hline $\begin{array}{l}\text { procesos de manera } \\
\text { independiente. Sólo se }\end{array}$ & $\begin{array}{l}\text { 2.4 Preparacion } \\
\text { de la mezcla }\end{array}$ & Fibra de vidrio & 0.70 & $\mathrm{~kg}$ & asociada al & emisiones por \\
\hline consideran los procesos a & & Ácido bórico & 0.70 & $\mathrm{~kg}$ & & electricidad. \\
\hline industriales llegan a planta. & & $\begin{array}{ll}\begin{array}{l}\text { Sulfato } \\
\text { potasio }\end{array} & \mathrm{de} \\
\end{array}$ & 3.48 & $\mathrm{~kg}$ & & \\
\hline & & Mezcla & 69.6 & $\mathrm{~kg}$ & & \\
\hline & 2.5 Fraguado & Papel reciclado & 10.4 & $\mathrm{~kg}$ & & \\
\hline & 2.6 Secado & Placa & 69.6 & $\mathrm{~kg}$ & & \\
\hline $\begin{array}{l}\text { 3. Comercialización } \quad y \\
\text { distribución }\end{array}$ & Celulosa & $\begin{array}{ll}\text { Papel con } & \text { con } \\
\text { adhesivo } & \\
\text { (etiqueta) } & \end{array}$ & 7.32 & $\mathrm{~m}$ & $\begin{array}{lr}\text { La } & \text { energía } \\
\text { asociada } & \text { al } \\
\text { proceso } & \text { de } \\
\text { empaque } & \text { y } \\
\text { combustibles. } & \\
\end{array}$ & $\begin{array}{l}\text { Gases } \\
\text { invernadero } \\
\text { por el uso de } \\
\text { combustibles. }\end{array}$ \\
\hline & & $\begin{array}{l}\text { Canal metálico } \\
\text { calibre } 26\end{array}$ & 2 & pza & & \\
\hline U Us lol nroduct & Jnctoloción & 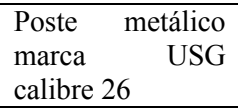 & 5 & pza & $\begin{array}{lr}\text { La } & \text { energía } \\
\text { asociada } & \text { al }\end{array}$ & Residuos de \\
\hline 4. Uso der producto & mstatacion & $\begin{array}{l}\text { Tornillos tipo } \mathrm{S}, \\
1 \text { " }\end{array}$ & 48 & pza & $\begin{array}{l}\text { proceso } \quad \text { de } \\
\text { instalación }\end{array}$ & instalación \\
\hline & & Pasta para juntas & 2.10 & $\mathrm{~kg}$ & & \\
\hline & & Cinta de refuerzo & 9.60 & $\mathrm{~m}$ & & \\
\hline & $\begin{array}{lr}5.1 & \text { Disposición } \\
\text { de } & \text { los } \\
\text { materiales } & \text { de } \\
\text { empaque } & \\
\end{array}$ & $\begin{array}{l}\text { Basura } \\
\text { doméstica: papel } \\
\text { con adhesivo }\end{array}$ & 7.32 & $\mathrm{~m}$ & $\begin{array}{ll}\text { La } & \text { energía } \\
\text { asociada } & \text { a los }\end{array}$ & Emisiones de \\
\hline 5. Fin de vida & & $\begin{array}{l}\text { Vertedero: } \\
\text { núcleo }\end{array}$ & 3 & pza & $\begin{array}{l}\text { procesos } \\
\text { recolección, }\end{array}$ & gases \\
\hline & $\begin{array}{rr}5.2 & \text { Disposición } \\
\text { del } & \text { tablero }\end{array}$ & $\begin{array}{l}\text { Vertedero: } \\
\text { Perfiles metálicos }\end{array}$ & 7 & pza & $\begin{array}{lr}\text { transporte } & \mathrm{y} \\
\text { tratamiento } & \mathrm{de}\end{array}$ & $\begin{array}{l}\text { asociados a la } \\
\text { descomposició }\end{array}$ \\
\hline & núcleo regular & $\begin{array}{l}\text { Vertedero: } \\
\text { Tornillos tipo S } \\
\text { 1" }\end{array}$ & 48 & $\mathrm{pz}$ & desechos. & \\
\hline
\end{tabular}

Fuente: Elaboración propia.: G. Madariaga, Delsordo N. Selene, González Q. A. Patricia (2014) 


\subsubsection{Transformación}

Impacto en los recursos materiales: los principales impactos se relacionan con la explotación de dichos recursos renovables y no renovables.

Impacto en los recursos energéticos: dentro de los procesos de obtención de materiales se produce un consumo energético, principalmente el de combustibles no renovables utilizados para la transportación de dichos materiales.

Impacto por emisiones tóxicas: cada categoría de materiales tiene impactos distintos en emisiones o residuos, pero son principalmente los asociados a la contaminación del aire por partículas y emisiones de gases invernadero.

\subsubsection{Comercialización y distribución}

Incluye los materiales y energía de distribución del producto terminado hacia los lugares físicos del canal de distribución, así como los materiales de protección y marca (etiquetas, empaque y embalaje). No se incluyen los materiales ni energía de traslado de componentes elementales en las diferentes etapas del proceso de extracción y transformación.

Impacto en los recursos materiales: en el caso de las Placas aligeradas, para su presentación al público, son atadas de dos en dos con una cinta de papel (base de celulosa) que se retira fácilmente al momento de ser instalada.

Impacto en los recursos energéticos: este es el rubro con mayor impacto en la etapa de comercialización y distribución, ya que las distancias pueden ser grandes, y por tanto, el consumo de combustibles también. También se incluye la energía requerida para el proceso de empaque individual y embalaje, que suele ser electricidad.

Impacto por emisiones tóxicas: el impacto es principalmente al aire, asociado con la emisión de gases invernadero, y en segundo lugar a los residuos de los materiales.

\subsection{Uso del producto}

Para esta etapa del ciclo de vida, sólo se consideran las actividades de instalación y mantenimiento del producto.

Impacto en los recursos materiales: los materiales que se agregan en esta etapa del ciclo de vida son los que conforman los accesorios de instalación del producto, como lo son: perfiles metálicos, tornillos, pasta y cinta.

Impacto en los recursos energéticos: se considera la energía asociada al proceso de instalación, la cual suele ser la energía eléctrica utilizada por aparatos como taladro.

Impacto por emisiones tóxicas: las emisiones de esta etapa son principalmente las de residuos de materiales de instalación.

\subsection{Fin de vida}

Los materiales de empaque y embalaje en su mayoría tienen un final de vida previo al producto, convirtiéndose en basura doméstica e industrial, dispuesta generalmente en vertederos. Cuando el producto en sí llega al final de vida, se convierte en un residuo, que es dispuesto como escombro.

Impacto en los recursos materiales: en esta etapa no se agregan recursos materiales relevantes. 
Impacto en los recursos energéticos: se considera el consumo de combustibles para la recolección, transporte y tratamiento de los desechos.

Impacto por emisiones tóxicas: principalmente el impacto consiste en la emisión de gases invernadero por el consumo de combustibles no renovables, y los asociados a la descomposición.

\subsubsection{Aplicación de Ecoindicadores}

Ahora, se multiplican por un indicador a través del programa Eco -it (Eco-it: 2014) las cantidades necesarias de materiales identificados en la Descripción de materiales En tablas (ejemplo, Tabla 2) se organiza esta información que cuantifica el impacto ambiental de un material o proceso de producción específico, lo cual muestra su utilidad al valorar diferentes estrategias de diseño. Para la aplicación de los indicadores se consideraron, en su mayoría, los materiales iniciales sin transformación. Algunas limitaciones a las evaluación y las tablas correspondientes se describen con detalle en el reporte de la ECC (reporte aún proceso de publicación) las limitaciones se deben principalmente a la disponibilidad de ecoindicadores más precisos.

\subsection{Valoración de Estrategias de Ecodiseño}

Al momento de escribir esto, las tablas restantes aunque muy avanzadas, aún se encuentran en la etapa de análisis y discusión, por lo cual se reportarán los detalles en documentos de investigación posteriores.

\section{Conclusiones}

Con la información disponible al momento de escribir esto se ofrecen algunas conclusiones preliminares:

Los resultados de la ECC obtenidos hasta ahora tienden a evidenciar un mejor comportamiento ambiental de las placas formuladas con pastas de yeso, partículas de plástico y fibra de agave, contra las placas comerciales empleadas para su evaluación.

Se puede concluir que los principales impactos al medio se encuentran en la obtención y procesado de los componentes, seguidos por su distribución y uso, mientras que la etapa de fin de vida reporta los menores impactos.

El modelo de ECC empleado, se ha mostrado como una herramienta de gran utilidad para los equipos diseñadores que buscan mejorar el desempeño ambiental de los productos sobre los que trabajan. Sus resultados potencian una mejor toma de decisiones en los equipos de desarrollo de productos.

La ECC es un proceso demandante en recursos humanos, técnicos y económicos esto coopera a que su aplicación sea incipiente en la industria.

La ECC es también una revisión de la calidad de las estrategias y productos evaluados, por lo cuál también puede ser aplicada como una herramienta para mejorar la competitividad de las empresas.

\section{Reconocimientos}

Nuestro reconocimiento a los jóvenes investigadores:

Zaira Selene Delsordo Núñez. Universidad Autónoma de Sinaloa. México.

Ana Patricia González Quiñonez. Inst. Tecnológico Superior de Cajeme, Sonora. México. 
Quiénes como jóvenes investigadores realizando una estancia en la Universidad de Guadalajara participaron activamente y de manera sustantiva en la realización de este trabajo. Mil gracias!

\section{Referencias bibliográficas}

GONZÁLEZ MADARIAGA, F.J. (2006) Caracterización de mezclas de residuos de poliestireno expandido conglomerados con yeso o escayola, su uso en la construcción. Tesis doctoral. UPC, ETSEIB. Barcelona ISBN 97884-690-6065-0

GONZÁLEZ MADARIAGA F.J \& LLOVERAS MACIÀ, J. (2008) EPS recycling bends mixed with plastic or stucco, some applications in building construction. Informes de la construcción.Instituto de Ciencias en la construcción Eduardo Torroja, Madrid. Issn: 0020-0883

GONZÁLEZ MADARIAGA F.J. \& ROSA SIERRA L.A. (2013) Caracterización de mezclas de residuos de poliestireno expandido (EPS) conglomerados con yeso, su uso en la construcción. Reporte de investigación 2008 2012. CUAAD. Universidad de Guadalajara, Guadalajara Jalisco, México. En preparación.

GONZÁLEZ MADARIAGA F.J (2012) Ecoeficiencia. Propuesta de diseño para el mejoramiento ambiental. Universidad de Guadalajara, Guadalajara Jalisco, México. ISBN 978607450679 2, México.

IHOBE (1999). ANEXO. Eco-indicator '99. Soc. Pública Gestión Medioambiental. Goedkoop et. al Bilbao

RIERADEVALL, J.\& VINYETS, J. (1999) Ecodisseny i ecoproductes. Barcelona: Rubes Editorial,S.L. 1a. Edición. 